\title{
Supporting Information: Rapid Structural, Kinetic and Immunochemical Analysis of Alpha-Synuclein Oligomers in Solution
}

\author{
William E. Arter ${ }^{1,2, \dagger}$, Catherine K. Xu ${ }^{1, \dagger}$, Marta Castellana-Cruz ${ }^{1}$, \\ Therese W. Herling ${ }^{1}$, Georg Krainer ${ }^{1}$, Kadi L. Saar ${ }^{1}$, \\ Janet R. Kumita, ${ }^{1}$ Christopher M. Dobson \& Tuomas P. J. Knowles ${ }^{1,2,{ }^{*}}$ \\ ${ }^{1}$ Department of Chemistry, University of Cambridge, Lensfield Rd, Cambridge, CB2 1EW UK \\ ${ }^{2}$ Cavendish Laboratory, University of Cambridge, JJ Thomson Avenue, Cambridge, CB3 OHE, UK \\ tThese authors contributed equally \\ 掝eced September 2019 \\ ${ }^{*}$ Corresponding author. Email tpjk2@cam.ac.uk
}

\section{Contents}

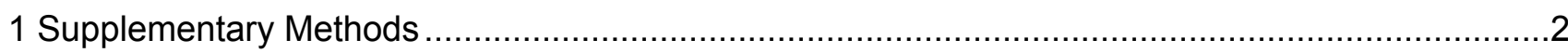

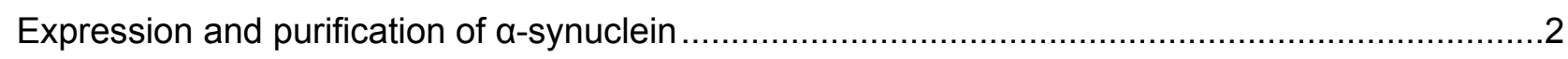

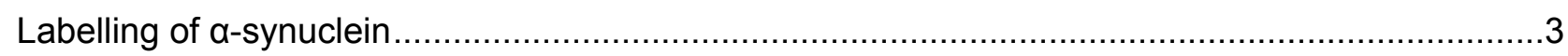

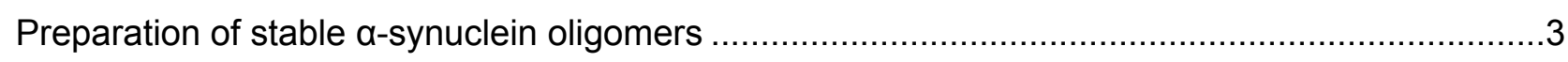

Procedure for generation and isolation of kinetic $\alpha$-synuclein oligomers .....................................

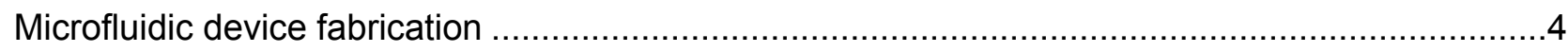

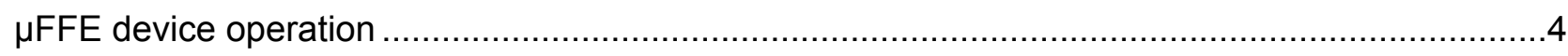

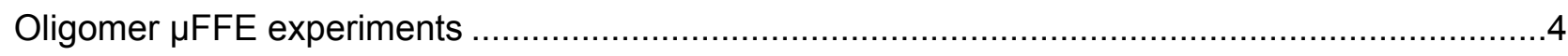

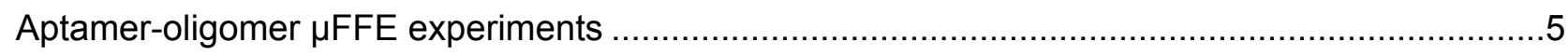

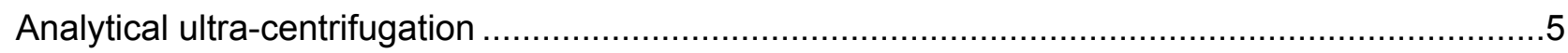

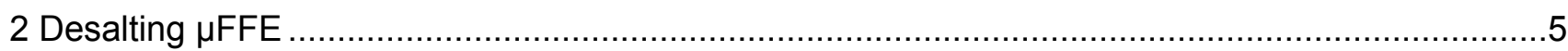

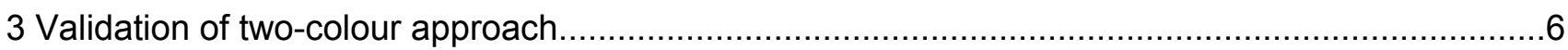

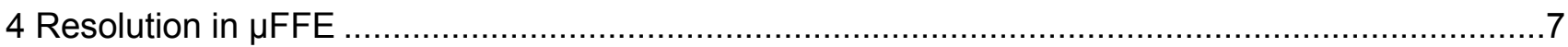

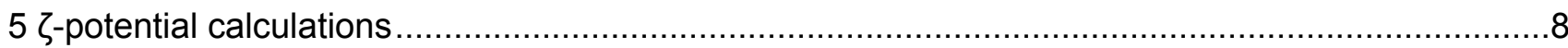

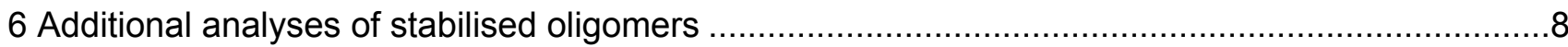

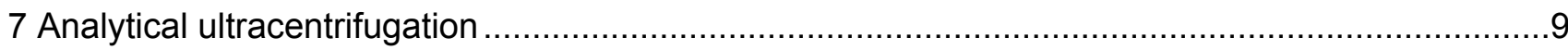

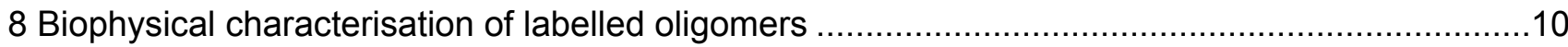

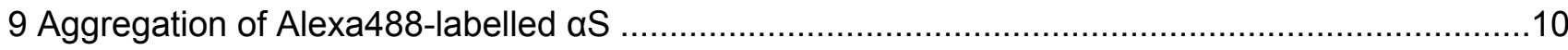

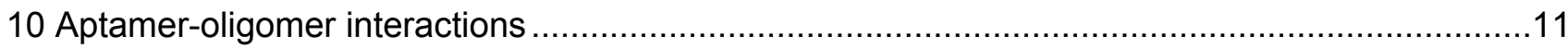

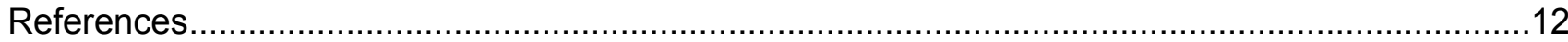




\section{Supplementary Methods}

\section{Expression and purification of $\alpha$-synuclein}

Wild-type $\alpha$-synuclein was expressed and purified as previously described. ${ }^{1}$ Briefly, Escherichia coli BL21 cells overexpressing $\alpha$-synuclein were collected by centrifugation ( $20 \mathrm{~min}, 4000 \mathrm{rpm}, 4^{\circ} \mathrm{C}$ ) in a JLA-8.1000 rotor in a Beckman Avanti J25 centrifuge (Beckman Coulter), resuspended in lysis buffer (10 mM Tris, $1 \mathrm{mM}$ EDTA, protease inhibitor), and lysed by sonication on ice. Following centrifugation (JA-25.5 rotor, $20 \mathrm{~min}, 18,000 \mathrm{rpm}, 4^{\circ} \mathrm{C}$ ), heat-sensitive proteins were precipitated out of the lysate supernatant by boiling, and subsequently removed by centrifugation (JA-25.5 rotor, 15 min, 18,000 $\left.\mathrm{rpm}, 4^{\circ} \mathrm{C}\right)$. DNA was precipitated out by incubation with streptomycin sulphate $(10 \mathrm{mg} / \mathrm{mL}, 15 \mathrm{~min}, 4$ ${ }^{\circ} \mathrm{C}$ ), and removed by centrifugation. $\alpha$-synuclein was precipitated out of the supernatant by the slow addition of ammonium sulphate $(361 \mathrm{mg} / \mathrm{mL})$ while stirring $\left(30 \mathrm{~min}, 4{ }^{\circ} \mathrm{C}\right)$. The pellet containing $\alpha$ synuclein was collected by centrifugation (JA-25.5 rotor, $15 \mathrm{~min}, 18,000 \mathrm{rpm}, 4^{\circ} \mathrm{C}$ ) and resuspended in $25 \mathrm{mM}$ Tris buffer, $\mathrm{pH} 7.4,20^{\circ} \mathrm{C}$. Following dialysis to ensure complete buffer exchange, the protein was loaded onto a HiLoadTM 26/10 Q Sepharose high performance column (GE Healthcare), and eluted at $\sim 350 \mathrm{mM} \mathrm{NaCl}, 20^{\circ} \mathrm{C}$ with a salt gradient from $0 \mathrm{M}$ to $1.5 \mathrm{M} \mathrm{NaCl}$. Selected fractions were subsequently loaded onto a Superdex $7526 / 60$ (GE Healthcare) at $20^{\circ} \mathrm{C}$ and eluted in PBS, pH 7.4, $20{ }^{\circ} \mathrm{C}$. Protein concentration was determined by absorbance at $275 \mathrm{~nm}$, using an extinction coefficient of $5600 \mathrm{M}^{-1} \mathrm{~cm}^{-1}$. The cysteine-containing variant (N122C) of $\alpha$-synuclein was purified by the same protocol, with the addition of $3 \mathrm{mM}$ DTT to all buffers.

\section{Labelling of $\alpha$-synuclein}

$\alpha$-Synuclein protein was fluorophore-labelled to enable visualisation by fluorescence microscopy. ${ }^{2}$ In order to remove DTT, cysteine variants of $\alpha$-synuclein were buffer exchanged into PBS or sodium phosphate buffer by use of P10 desalting columns packed with Sephadex G25 matrix (GE Healthcare). The protein was then incubated with an excess of Alexa488 or Alexa546 dyes with maleimide moieties (Thermofisher Scientific) (overnight, $4{ }^{\circ} \mathrm{C}$ on a rolling system) at a molar ratio of 1:1.5 (protein-to-dye). The labelling mixture was loaded onto a Superdex 200 16/600 (GE Healthcare) and eluted in PBS or $10 \mathrm{mM}$ sodium phosphate $\mathrm{pH} 7.4$ buffer at $20^{\circ} \mathrm{C}$, to separate the labelled protein from free dye. The concentration of the labelled protein was estimated by the absorbance of the fluorophores, assuming a 1:1 labelling stoichiometry (Alexa488: $72000 \mathrm{M}^{-1} \mathrm{~cm}^{-1}$ at $495 \mathrm{~nm}$, Alexa546: $112000 \mathrm{M}^{-1} \mathrm{~cm}^{-1}$ at $\left.556 \mathrm{~nm}\right)$. 


\section{Preparation of stable $\alpha$-synuclein oligomers}

a-synuclein oligomers were prepared by the following procedure. ${ }^{2}$ Monomeric $\alpha$-synuclein was lyophilised in Milli-Q water and subsequently resuspended in PBS or $10 \mathrm{mM}$ sodium phosphate buffer, both at $\mathrm{pH} 7.4$, to give a final concentration of ca. $840 \mathrm{uM}(12 \mathrm{mg} / \mathrm{mL})$. The resulting solution was passed through a $0.22 \mathrm{uM}$ cut-off filter before incubation at $38{ }^{\circ} \mathrm{C}$ for $20-24 \mathrm{~h}$ under quiescent conditions. Very small amounts of fibrillar species formed during this time were removed by ultracentrifugation for $1 \mathrm{~h}$ at $90000 \mathrm{rpm}$ (Optima TLX Ultracentrifuge, Beckman Coulter, TLA-120.2 Beckman rotor, $288000 \mathrm{~g}$ ). The excess monomeric protein and some small oligomers were then removed by multiple filtration steps using $100-\mathrm{kDa}$ cut-off membranes. The final concentration of oligomers was estimated by the absorbance at $495 \mathrm{~nm}$ using a molar extinction coefficient of 72000 $\mathrm{M}^{-1} \mathrm{~cm}^{-1}$ for the Alexa488 labelled oligomers.

\section{Procedure for generation and isolation of kinetic $\alpha$-synuclein oligomers}

Alexa488 labelled $\alpha$-synuclein (N122C, $100 \mu \mathrm{M}, 500-800 \mu \mathrm{L})$ was incubated in PBS buffer, pH 7.4, at $37{ }^{\circ} \mathrm{C}$ with $0.01 \%$ sodium azide, in a $1.5 \mathrm{~mL}$ Eppendorf tube with shaking at $200 \mathrm{rpm}$ over 96 hours. $150 \mu \mathrm{L}$ aliquots were withdrawn and centrifuged for $15 \mathrm{~min}$ at $21130 \mathrm{~g}$, to pellet insoluble, fibrillar components of the reaction mixture. The supernatant, containing monomeric and oligomeric $\alpha$ synuclein, was carefully removed and used immediately for FFE experiments. A small portion of the supernatant was retained for analysis of $\alpha$-synuclein concentration by UV-vis absorption spectroscopy.

\section{Microfluidic device fabrication}

Devices were designed using AutoCAD software (Autodesk) and photolithographic masks printed on acetate transparencies (Micro Lithography Services). Polydimethylsiloxane (PDMS) devices were produced on SU-8 moulds fabricated via photolithographic processes as described elsewhere, ${ }^{3,4}$ with UV exposure performed with custom-built LED-based apparatus. ${ }^{5}$ Following development of the moulds, feature heights were verified by profilometer (Dektak, Bruker) and PDMS (Dow Corning, primer and base mixed in 1:10 ratio) applied and degassed before baking at $65^{\circ} \mathrm{C}$ overnight. Devices were cut from the moulds and holes for tubing connection $(0.75 \mathrm{~mm})$ and electrode insertion $(1.5 \mathrm{~mm})$ were created with biopsy punches, the devices were cleaned by application of Scotch tape and sonication in IPA (5 min). After oven drying, devices were bonded to glass slides using an oxygen plasma. Before use, devices were rendered hydrophilic via prolonged exposure to oxygen plasma. ${ }^{6}$ 


\section{$\mu F F E$ device operation}

Liquid-electrode microchip free-flow electrophoresis ( $\mu \mathrm{FFE}$ ) devices were operated as described previously. ${ }^{7}$ Briefly, fluids were introduced to the device by PTFE tubing, 0.012"ID x 0.030"OD (ColeParmer) from glass syringes (Gas Tight, Hamilton) driven by syringe pumps (Cetoni neMESYS). $\mu$ FFE experiments were conducted with auxiliary buffer, electrolyte, monomer reference and sample flow rates of $1200,250,140$ and $10 \mu \mathrm{L} \mathrm{h}^{-1}$, respectively, for $15 \times$ reduction in buffer salt concentration for samples in PBS buffer.

Potentials were applied by a programmable benchtop power supply (Elektro-Automatik EA-PS 950006) via bent syringe tips inserted into the electrolyte outlets. Device voltage efficiency was calibrated by comparison of current-voltage curves of the device operating under assay conditions and when filled with $3 \mathrm{M} \mathrm{KCl}$ electrolyte. Efficiencies were found to be $\approx 20 \%$, affording electric fields equivalent to $200-267 \mathrm{~V} \mathrm{~cm}^{-1}$ for potentials of $300-400 \mathrm{~V}$.

Microfluidic experiments were conducted using an inverted fluorescence microscope (Zeiss AxioObserver D1), Alexa488, 546 and 647-labelled species were observed using appropriate filter sets (49002, 49004 and 49006, Chroma Technology) and camera (Evolve 512 CCD, Photometrics). Control experiments confirmed negligible spectral overlap between the filter sets employed.

\section{Oligomer $\mu F F E$ experiments}

Alexa488-labelled oligomeric mixtures ( $4 \mu \mathrm{M}$ monomer equivalent for stabilised oligomers) in either $10 \mathrm{mM}$ sodium phosphate pH 7.4 or PBS buffer were mixed on-chip with Alexa546-labelled monomer $(2 \mu \mathrm{M})$ in either $10 \mathrm{mM}$ sodium phosphate $\mathrm{pH} 7.4$ or pure water, respectively. For oligomeric samples in $10 \mathrm{mM}$ sodium phosphate or PBS buffer, auxiliary buffer comprised of the same or 15X diluted PBS buffer, respectively, supplemented with $0.05 \%$ v/v Tween-20.

Following data acquisition, Alexa488 and Alexa546 fluorescence profiles were extracted by taking a line profile (100-pixel thick) perpendicular to the direction of flow using ImageJ software. The fluorescence profiles were superimposed and normalised to the maximum peak fluorescence of the Alexa546 monomer. This operation enabled visualisation and subsequent subtraction of the monomer-only component of the monomer+oligomer mixture visible in the 488 channel, and accounted for differences in monomer fluorescence intensity between the 488 and 546 channels which occur due to differences in protein concentration and in the characteristics of the fluorophores. This process resulted in almost identical 488 and 546 fluorescence profiles for the monomer portion of the combined electropherogram (Fig1(d, e), main text).

Subtraction of the normalised Alexa546 from the Alexa488 profile afforded profiles due to oligomeric aggregates alone. For quantification of oligomers formed transiently during aS aggregation, the 
relative peak integrals of oligomeric and monomer populations were compared to the known monomer concentration and degree of sample loss during desalting (see below) to calculate the oligomer concentration.

\section{Aptamer-oligomer $\mu \mathrm{FFE}$ experiments}

Wild-type aS $(100 \mu \mathrm{M})$ was mixed with Alexa647-labelled aptamer $(2 \mu \mathrm{M}$, Integrated DNA Technologies, sequence is provided in the Supporting Information) in PBS buffer and underwent aggregation by rapid stirring. ${ }^{8} 150$ uL aliquots were withdrawn every hour and fibrillar material was removed by centrifugation. The sample then underwent desalting- $\mu$ FFE analysis as described above.

\section{Analytical ultra-centrifugation}

Sedimentation velocity measurements ${ }^{9}$ were carried out at $20^{\circ} \mathrm{C}, 43000 \mathrm{rpm}(136680 \mathrm{~g})$, using a Beckman Coulter Optima XL-1 analytical ultracentrifuge equipped with UV-visible absorbance optics and an AN50-Ti rotor. The sedimentation coefficient distributions, corrected to standard conditions by using the SEDNTERP programme, were calculated via least-squares boundary modelling of sedimentation velocity data using the $\mathrm{c}(\mathrm{s})$ and $\mathrm{Is}-\mathrm{g}^{*}(\mathrm{~s})$ methods, as implemented in the SEDFIT programme. $^{10}$

\section{Desalting $\mu F F E$}

To increase the applicability of our method towards the analysis of oligomeric samples in general, including under physiological conditions, we sought to remove the constraints regarding buffer type that are a feature of other electrophoresis experiments. $\mu \mathrm{FFE}$ fractionation requires low-salt conditions (e.g. typically $<20 \mathrm{mM} \mathrm{NaCl}$ ), often facilitated through the use of non-physiological buffering agents, ${ }^{11}$ in order to minimise ionic conduction which can otherwise severely limit the electric field that can be induced across the electrophoresis chamber. This is problematic for the analysis of samples in high-salt, physiological buffers such as PBS, and is particularly relevant in the context of protein aggregation given the effect of solution conditions on oligomer stability and formationdissociation kinetics. ${ }^{12,13}$

A desalting module was incorporated into the $\mu F F E$ device, upstream of the electrophoresis chamber, to rapidly decrease the salt concentration on chip to enable electrophoretic analysis (Figure S1).

The desalting module functions by exploiting the relatively slow rate of diffusion for high molecular weight proteins and protein oligomers in comparison to that of salt ions. Sample solutions undergo diffusional mixing with salt-free water during passage through the desalting module, salts diffuse the full width of the microfluidic channel and are diluted according to the relative flow rates of sample and 
diluent, whilst proteins only partially diffuse and remain at higher concentration before passage into the electrophoresis chamber. The protein is then re-collected on-chip, before passing into the electrophoresis chamber of the device for $\mu \mathrm{FFE}$ analysis. Although a proportion of the sample is lost using this technique, this effect is minimised for species with slow diffusion such as the large oligomeric complexes of interest here. The degree of sample loss is shown in Figure $S 1(c, d)$, with $47 \%$ of monomer $(r=3 \mathrm{~nm})$ retained for electrophoresis, increasing to $65-75 \%$ for oligomers of $r=$ $6.4-10 \mathrm{~nm} .^{2}$ Ionic strength can be readily reduced by up to $20 \times$, bringing samples initially in PBS into suitable operating conditions for effective $\mu \mathrm{FFE}$. Importantly, this desalting process occurs quickly, with the initial desalting step and total time on chip being $\sim 1.8$ and 3.5 seconds, respectively. Therefore, it is predicted that desalting will not significantly affect oligomer structure over the assay timescale, which is significant since solution conditions are known to influence aggregate stability. ${ }^{12}$ In addition, the monomer protein reference can be co-injected with the salt-free diluent, minimising the time for potential interactions between reference and analyte.

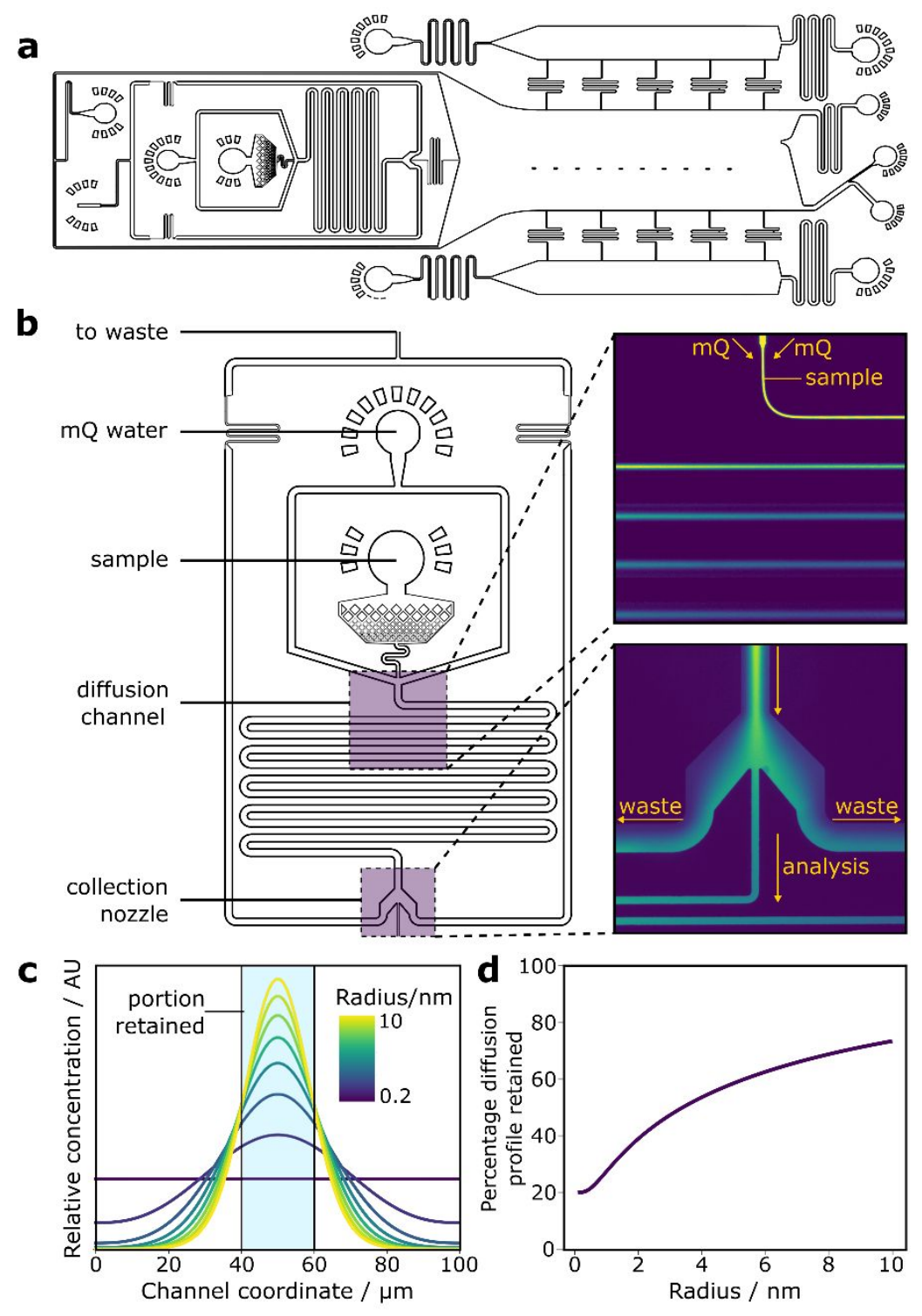


Figure S1 $\mu$ FFE device with in-line desalting module. (a) Schematic of desalting- $\mu F F E$ device design. (b) Enlarged schematic of the desalting module. (Inset, upper) Fluorescence micrograph of aS monomer flowing into the desalting-diffusion region. (Inset, lower) Fluorescence micrograph of desalting 'nozzle', showing central portion of aS monomer diffusion profile being selected for downstream $\mu F F E$ analysis. (c) Schematic showing region of diffusion profiles for $r=0.2 \mathrm{~nm}\left(\mathrm{Na}^{+}\right.$ ions) to $r=10 \mathrm{~nm}$ (approximate size of $\alpha \mathrm{S}$ oligomers). (d) Plot of percentage of protein retained for downstream analysis as a function of hydrodynamic radius.

\section{Validation of two-colour approach}

For the dual-colour approach described here, it is necessary that both Alexa488 and Alexa546 labelled aS possess the same electrophoretic properties. Since both dyes possess the same charge $(-2$ e) and a similar structure, the difference between the labelling variants was expected to be minimal. To verify this prediction, the electrophoretic mobilities of monomeric Alexa488 and Alexa546 labelled aS were quantified using previously described methods (Figure S2). ${ }^{7}$ From microscopy images of electrophoretic deflection at the two label wavelengths, no difference could be observed between the two labelling variants (Figure $S 2(b, c)$ ). To assess this finding quantitatively, the electrophoretic mobilities of the variants were found by plotting the electrophoretic drift velocity as a function of applied electric field. From the linear relationship obtained, it was clear that the measured electrophoretic mobility of $\mu=-1.43 \pm 0.11 \times 10^{-8} \mathrm{~m}^{2} \mathrm{~V}^{-1} \mathrm{~s}^{-1}$ was the same for both fluorophores within experimental error. 


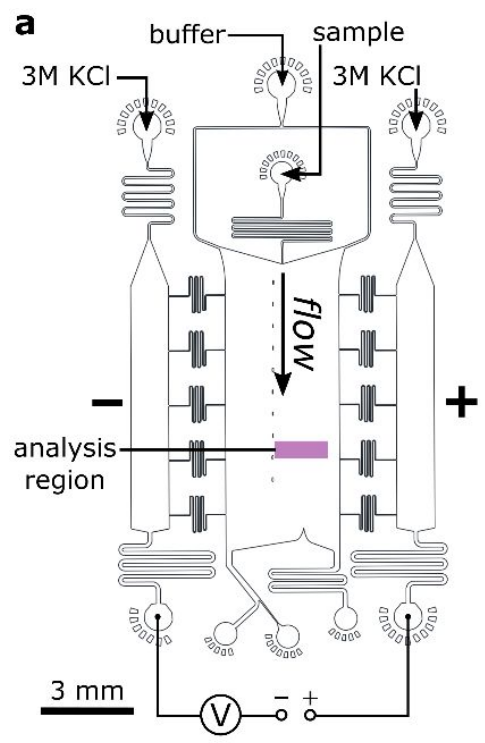

b

Alexa-488 Alexa-546 Merged
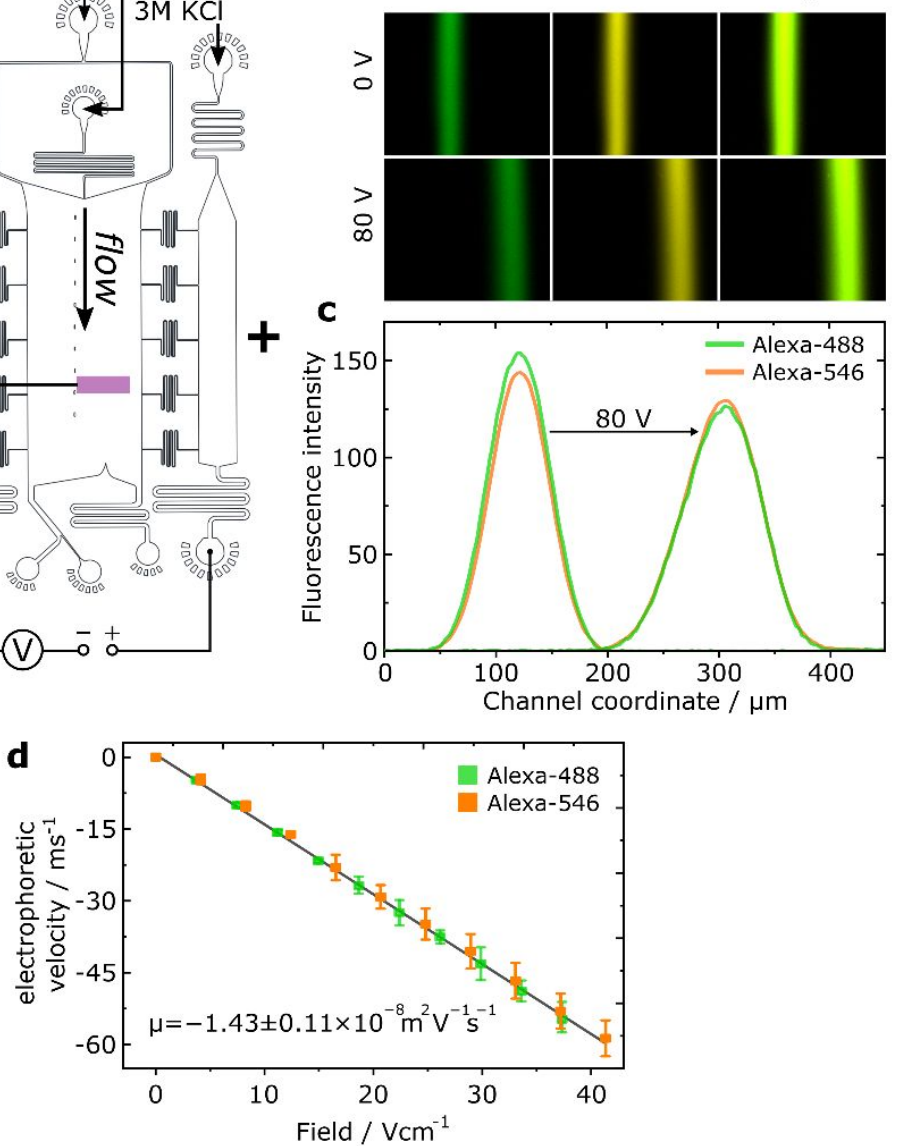

Figure S2 Quantitative FFE of monomeric aS. (a) Design with annotated fluid inlets for the microfluidic FFE device used for quantification of monomeric $\alpha S$ and oligomeric $\alpha S$ in low-salt buffer. (b) Microscopy images of Alexa488, Alexa546 and merged fluorescence of labelled aS monomer, mixed in the same sample and imaged using orthogonal excitation and emission filters, at $0 \mathrm{~V}$ and 80 V. (c) Superimposed electropherograms of Alexa488 and Alexa546 labelled aS at $0 \mathrm{~V}$ and $80 \mathrm{~V}$ corresponding to images shown in (b). (d) Scatter plot and linear fit of Alexa488 and Alexa546 electrophoretic velocities as a function of applied field. Both labelling variants display the same electrophoretic mobility within experimental error.

\section{Resolution in $\mu \mathrm{FFE}$}

To account for the finite resolution of the electrophoresis technique, we describe electrophoresis profiles in terms of 'apparent mobility'. Importantly, the mobilities of discrete electropherograms peaks are reported accurately in terms of their absolute value, but there exists an inherent uncertainty for electropherogram positions between intensity maxima. This uncertainty is given by the width of the sample beam under electrophoresis, the variance of which is approximated by: ${ }^{14}$ 


$$
\sigma_{\text {total }}^{?}=\sigma_{i n j}^{?}+\sigma_{D}^{?}+\sigma_{H D}^{?}=\frac{w_{i n j}^{?}}{12}=\frac{2 D L}{v}+\frac{h h^{2} d^{2} v}{105 D L}
$$

where $\sigma_{i n j}^{?}, \sigma_{D}^{?}$ and $\sigma_{H D}^{?}$ describe the injection width, diffusional and hydrodynamic contributions to the total beam width. $W_{i n j}$ is the width of the sample injection stream, and $v, D, L, d$, and $h$ are the linear flow velocity, diffusion coefficient, channel length beam deflection and channel height, respectively. For nominal oligomer and monomer radii of $6.4 \mathrm{~nm}$ and $3 \mathrm{~nm},{ }^{15,16}$ values of $\sigma_{\text {total }}=176$ $\mu \mathrm{m}$ and $80 \mu \mathrm{m}$ were calculated for the flow conditions and device geometry used here. Although these values correspond to $28 \%$ and $25 \%$ of the respective total deflection for oligomer and monomer, an important factor to note is that this band-broadening effect does not prohibit the observation of discrete peaks in oligomer electrophoresis profiles. Thus, the finite resolution of $\mu F F E$ does not preclude observation and analysis of oligomer heterogeneity.

\section{$5 \quad \zeta$-potential calculations}

$\zeta$-potentials are typically calculated for colloidal systems where $\kappa r \gg 1$ using the Smoluchowski relation $\mu=\frac{\epsilon_{0} \epsilon_{r} \zeta}{\eta}$ where $\kappa$ is the inverse Debye length and $r$ is the particle radius. However, using the most common oligomer radius of $r_{o}=6.4 \mathrm{~nm}$ as determined by AUC, ${ }^{2,15}$ and since $\kappa=0.52 \mathrm{~nm}$ 1 in our experiment (10 mM NaPi), $\kappa r \approx 3.3$ and the Smoluchowski model is therefore an unsuitable model for our data. Instead, the Henry equation: ${ }^{17}$

$$
\zeta=\frac{3 \mu \eta}{2 \epsilon_{0} \epsilon_{r} f_{H}(\kappa a)}
$$

provides a better approximation, where $f_{H}$ is the Henry function, approximated as: ${ }^{17}$

$$
\begin{gathered}
f_{H}(\kappa a)=1+\frac{1}{2(1+\delta)^{3}} \\
\delta=\frac{5}{2 \kappa a\left(1+2 e^{-\kappa a}\right)}
\end{gathered}
$$

from these equations, values of $\delta=0.7$ and $f_{H}(\kappa a)=1.1$ were obtained for $r=6.4 \mathrm{~nm}$. Thus, a value of $\zeta=-42.6 \pm 4.1 \mathrm{mV}$ was obtained for the largest population of oligomers where $\mu=-2.49 \pm$ $0.16 \times 10^{-8} \mathrm{~m}^{2} \mathrm{~V}^{-1}$ (main text, Figure $1(\mathrm{f})$ ).

\section{Additional analyses of stabilised oligomers}

Figure S3 shows additional data for $\mu F F E$ and AUC analysis of oligomer heterogeneity, from oligmomeric mixtures synthesised in either $10 \mathrm{mM}$ sodium phosphate or PBS buffer. For the NaPi sample, a different oligomer profile is observed compared to the analysis described in the main text, 
due to differences between samples caused by small variances in oligomer preparation. Importantly, the AUC and $\mu F F E$ data is self-consistent within each sample dataset, confirming the efficacy of our approach.

In the case of the PBS samples, clear separation between the oligomer and monomer peaks demonstrates effective sample preparation by the microfluidic desalting unit. By comparing the data between the NaPi and PBS-buffered samples, a greater concentration of monomeric aS is present in the lower-salt conditions. This is due to a greater degree of sample dissociation occurring under low ionic strength during the time between oligomer preparation and measurement $(2-4 \mathrm{~h}) .{ }^{12}$ Notably, the monomeric fraction is minimal in comparison to the oligomer peak observed in the $\mu$ FFE data for PBSbuffered oligomers. This implies that no appreciable dissociation of oligomers occurs during the timescale of the $\mu F F E$ experiment.
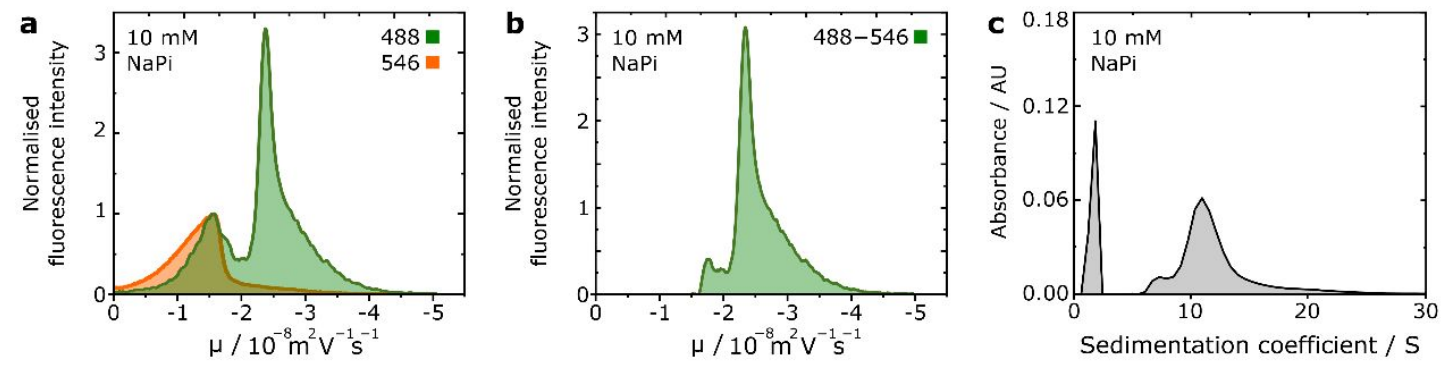

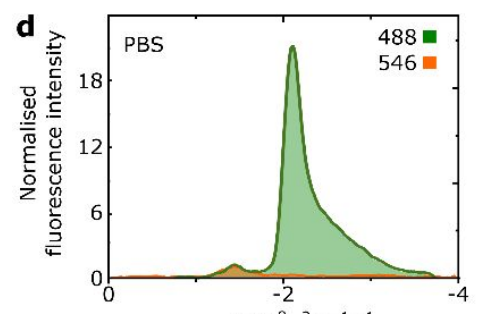

$\mu / 10^{-8} \mathrm{~m}^{2} \mathrm{v}^{-1} \mathrm{~s}^{-1}$

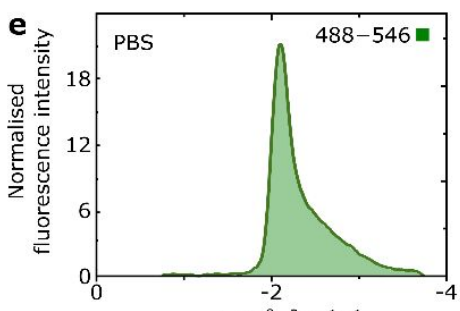

$\mu / 10^{-8} \mathrm{~m}^{2} \mathrm{~V}^{-1} \mathrm{~s}^{-1}$

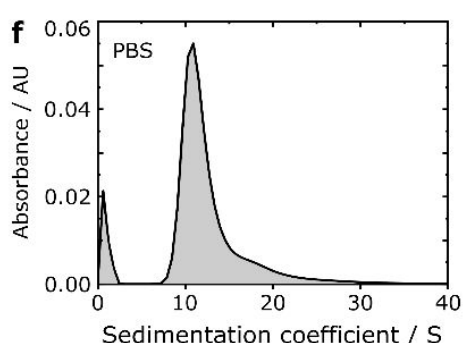

Sedimentation coefficient / S

Figure S3 Additional $\mu F F E$ and AUC data for synthetic oligomers. (a) Superimposed electropherograms for monomer-normalised Alexa546 labelled monomer and Alexa488 labelled oligomer sample in $10 \mathrm{mM} \mathrm{NaPi}$ buffer. (b) Electropherogram corresponding to only the oligomeric fraction of an oligomer sample in $10 \mathrm{mM} \mathrm{NaPi}$ buffer. (c) AUC data for the same oligomer sample as in (a) and (b), the peak at $S \approx 2$ is due to monomeric protein. (d) Superimposed electropherograms for $\mu \mathrm{FFE}$ of monomer-normalised Alexa546 labelled monomer and Alexa488 labelled oligomer sample desalted on chip from PBS buffer. (e) Electropherogram corresponding to only the oligomeric fraction of an oligomer sample desalted on chip from PBS buffer. (f) AUC data for the same oligomer sample as in (d) and (e), the peak at $S \approx 2$ is due to monomeric protein.

\section{Analytical ultracentrifugation}


To obtain an independent measure of structural heterogeneity, AUC experiments were conducted using the same two samples of stable aS oligomers. AUC quantifies the sedimentation coefficient $(s)$ of particles, defined as the ratio of a particle's sedimentation velocity to the applied acceleration that causes the sedimentation. $s$ is given by:

$$
s=\frac{m}{6 \pi \eta r}
$$

where $m$ and $r$ are the mass and radius of the particle. For oligomers, the mass of particles will share a linear relationship with the degree of oligomerisation $n_{m}$, which is in turn related to the net charge and electrophoretic mobility according to:

$$
\begin{gathered}
\mu_{o} \propto \frac{q_{o}}{r_{o}} \propto \frac{n_{m}^{\prime \prime}}{r_{o}} \\
n_{m} \approx \frac{V_{o}}{V_{m}}=\frac{r_{o}^{2}}{r_{m}^{2}} \\
r_{o}=r_{m} n_{m}^{\frac{1}{3}} \\
\mu_{o} \propto \frac{n_{m}^{\nu}}{r_{m} n_{m}^{\frac{1}{3}}} \propto \frac{n_{m}^{\nu}}{r_{m}}
\end{gathered}
$$

Therefore, the sedimentation coefficient may scale with oligomer size in a similar manner to electrophoretic mobility according to:

$$
\begin{gathered}
s_{o} \propto \frac{m_{o}}{r_{o}} \propto \frac{n_{m}}{r_{o}} \\
\mu_{o} \propto s_{o}^{\prime *}
\end{gathered}
$$

where $s_{o}$ and $m_{o}$ are the sedimentation coefficient and mass of an oligomer of $n_{m}$ monomer units, respectively. Therefore, similar observations of oligomer heterogeneity are expected between $\mu F F E$ and AUC, though these measurements are not directly proportional due to the scaling exponent $0<v<1$, that links net oligomer charge to $n_{m}$.

Oligomer hydrodynamic radii were approximated from AUC data by calculation of oligomer mass as a function of oligomer size, assuming spherical oligomers and protein density ${ }^{18} \rho=1.35 \mathrm{gcm}^{-1}$ according to:

$$
S=10^{-13} S=\frac{V_{o} \rho}{6 \pi \eta r_{o}}
$$




\section{Biophysical characterisation of labelled oligomers}
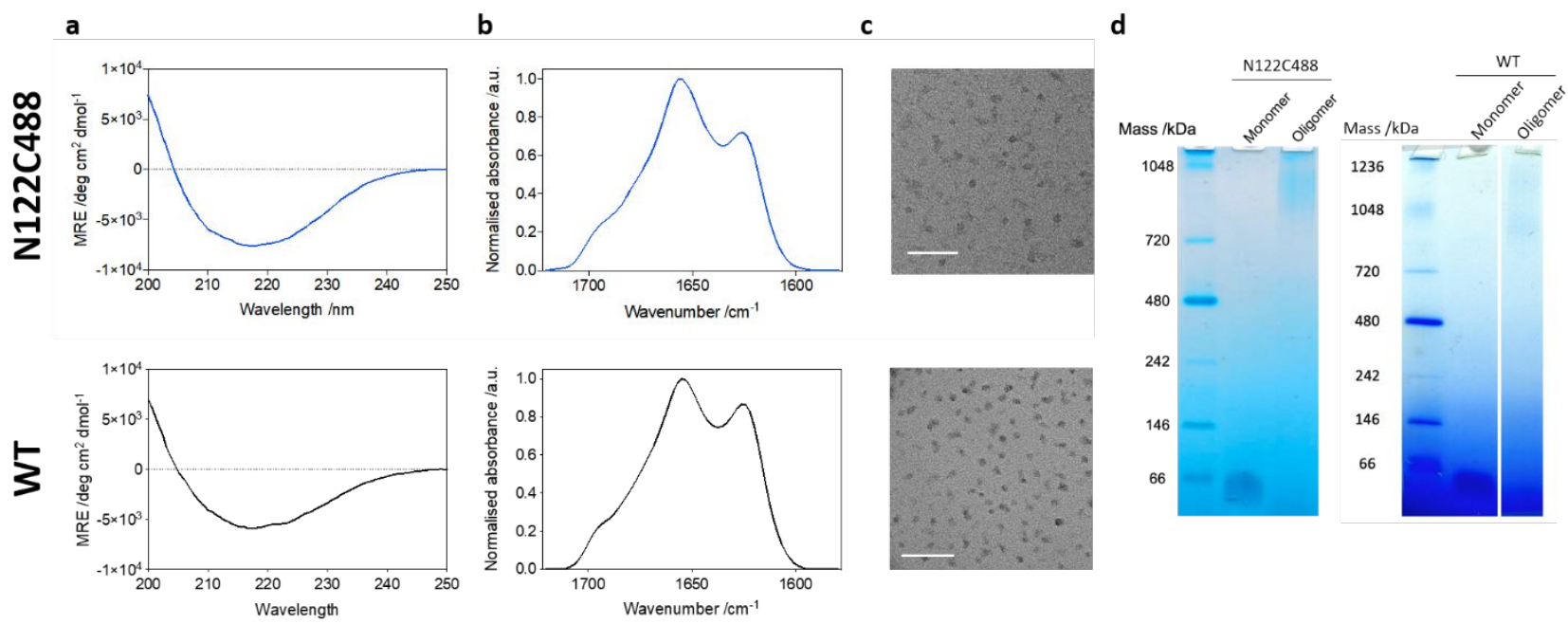

Figure S4 Biophysical characterisation of Alexa488-labelled and wild-type stable aS oligomers. (a) Representative circular dichroism (CD) spectra of oligomers formed of Alexa488 labelled N122C (upper) and wild-type (lower) alpha-synuclein. (b) Representative Fourier-transform infra-red spectroscopy (FTIR) spectra of oligomers formed of Alexa488 labelled N122C (upper) and wild-type (lower) alpha-synuclein, showing the expected antiparallel $\beta$-sheet structural content. $^{2}$ (c) Transmission electron microscope (TEM) image of oligomers formed of Alexa488 labelled N122C (upper) and wild-type (lower) alpha-synuclein (scale bar $=100 \mathrm{~nm}$ ). (d) Gel electrophoresis of Alexa488 labelled N122C monomer and oligomer (left) and wild-type (WT) aS monomer and oligomer (right). The large apparent mass of monomeric aS (true mass $\approx 14 \mathrm{kDa}$ ) is ascribed to the intrinsically disordered properties of $\alpha \mathrm{S}$, as opposed to the globular proteins of the marker ladder. Further comparison, including atomic force microscopy (AFM) analysis between wild-type and labelled aS oligomer can be found in previously published work. ${ }^{2}$ 


\section{Aggregation of Alexa488-labelled aS}

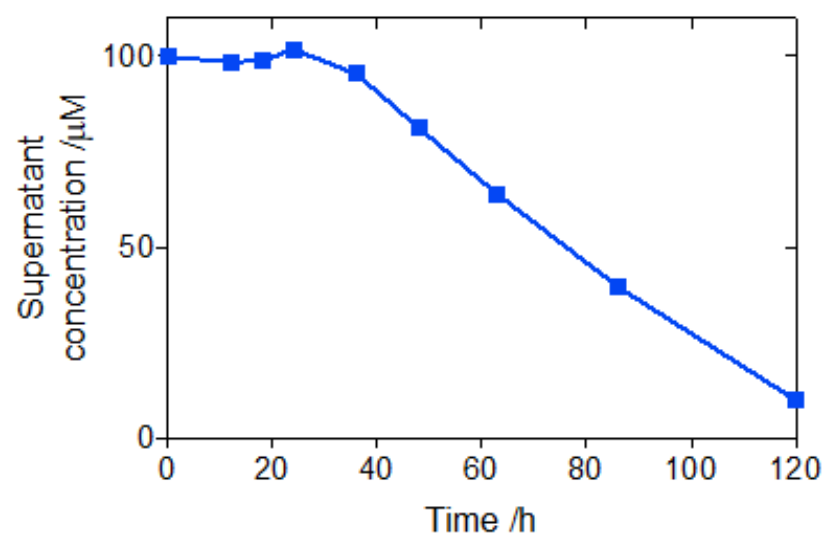

Figure 5 Aggregation kinetics of Alexa488-labelled $\alpha S$ under shaking (200 rpm) at $37^{\circ} \mathrm{C}, \mathrm{PBS} \mathrm{pH}$ 7.4. Fibrils were removed by centrifugation (21,130 rcf, $\left.10 \mathrm{~min}, 25{ }^{\circ} \mathrm{C}\right)$ and the supernatant concentration quantified by UV-Vis spectroscopy at $495 \mathrm{~nm}$.

\section{Aptamer-oligomer interactions}

The aptamer used here (Table S1) is known to be strongly selective for oligomers, but also weakly binds fibrillar $\alpha S .{ }^{19}$ To exclude the possibility of the lower-mobility band observed in the $4.25 \mathrm{~h} \alpha \mathrm{S}$ aggregration timepoint being due to aptamer binding to erroneously retained fibrillar material, $\mu \mathrm{FFE}$ was conducted with a sample mixture comprising aptamer and fibrillar aS (Figure S6(a, b)). A clear difference in electrophoretic mobility and electropherogram profile was observed for the aptamer band, in comparison to both the $\mathrm{t}=0$ and $\mathrm{t}=4.25 \mathrm{~h}$ timepoints. This indicates that fibrillar material may also be detectable by $\mu \mathrm{FFE}$, as suggested previously, ${ }^{7}$ and that the band observed at $\mathrm{t}=4.5 \mathrm{~h}$ can be assigned to aptamer-oligomer binding.

Samples of aS fibrils were prepared as described previously. ${ }^{2}$ Briefly, monomeric $\alpha S$ at $70 \mu \mathrm{M}$ in PBS buffer $\mathrm{pH} 7.4,0.01 \% \mathrm{NaN}_{3}$, was incubated under constant agitation ( $37^{\circ} \mathrm{C}, 200 \mathrm{rpm}$ ) for $4-6$ days. After this time, each sample was centrifuged (15 min at $13200 \mathrm{rpm}$ ) and the fibrillar pellet washed 
twice with PBS before being resuspended into the appropriate volume of PBS. The final concentration of fibrils, that was typically ca. $100 \mu \mathrm{M}$ in each sample, was estimated by measuring the absorbance at $275 \mathrm{~nm}$ using a molar extinction coefficient of $5600 \mathrm{M}^{-1} \mathrm{~cm}^{-1}$ after disaggregating an aliquot in the presence of $4 \mathrm{M}$ guanidinium chloride. To reduce their size to $\approx 100 \mathrm{~nm}$ to prevent blockage of device channels, fibrils were sonicated (20s, $10 \%$ power, $30 \%$ cycle) prior to use. Aptamer-fibril $\mu F F E$ was conducted in PBS buffer $\mathrm{pH} 7.4$, with aptamer and fibril (monomer equivalent) concentrations of $2 \mu \mathrm{M}$ and $100 \mu \mathrm{M}$, respectively.
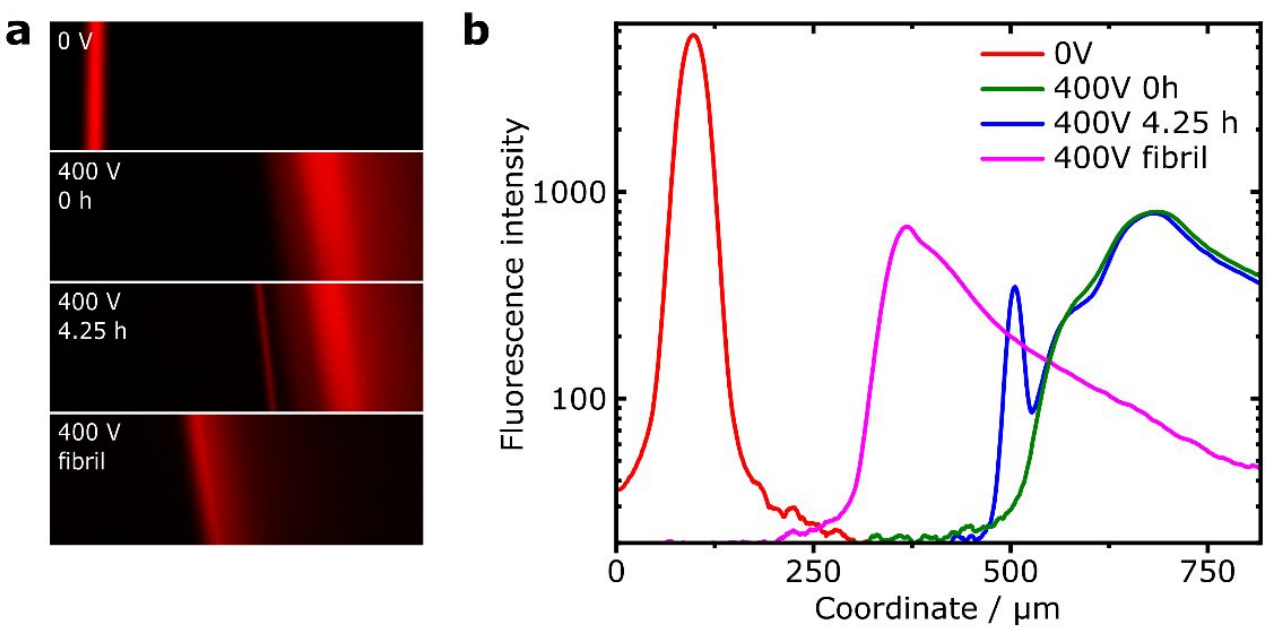

Figure S6 (a, upper three panels) Microscopy images of aptamer fluorescence during $\mu F F E$, from aptamer mixed into an aggregation reaction of wild-type $\alpha S$, as in the main text. (Bottom panel) $\mu F F E$ of an aptamer-fibril mixture, using sonicated fibrils of wild-type aS. (b) Electrophoresis profiles of aptamer $\mu F F E$, as in main text, with addition of profile for aptamer-fibril mixture.

\begin{tabular}{|c|c|}
\hline $\begin{array}{c}\text { DNA } \\
\text { oligonucleotide }\end{array}$ & Sequence \\
\hline $\begin{array}{c}\text { aS oligomer- } \\
\text { selective aptamer }\end{array}$ & 5'-(Alexa647)-ATTTGCCTGTGGTGTTGGGGCGGGTGCG-3' \\
\hline
\end{tabular}

Table S1 DNA sequence for aptamer selective for aS oligomers. Aptameric sequence T-SO508 is underlined. ${ }^{19}$ Additional four bases were added to provide spacer between aptameric region and fluorophore. 


\section{References}

1. Hoyer, W. et al. Dependence of $\alpha$-Synuclein Aggregate Morphology on Solution Conditions. J. Mol. Biol. 322, 383-393 (2002).

2. Chen, S. W. et al. Structural characterization of toxic oligomers that are kinetically trapped during a-synuclein fibril formation. Proc. Natl. Acad. Sci. U. S. A. 112, E1994-2003 (2015).

3. Mazutis, L. et al. Single-Cell Analysis and Sorting Using Droplet-Based Microfluidics. Nat. Protoc. 8, 870-891 (2013).

4. McDonald, J. C. et al. Fabrication of Microfluidic Systems in Poly(dimethylsiloxane). Electrophoresis 21, 27-40 (2000).

5. Challa, P. K., Kartanas, T., Charmet, J. \& Knowles, T. P. J. Microfluidic Devices Fabricated Using Fast Wafer-Scale LED-Lithography Patterning. Biomicrofluidics 11, 014113 (2017).

6. Tan, S. H., Nguyen, N.-T., Chua, Y. C. \& Kang, T. G. Oxygen Plasma Treatment for Reducing Hydrophobicity of a Sealed Polydimethylsiloxane Microchannel. Biomicrofluidics 4, 32204 (2010).

7. Saar, K. L. et al. On Chip Label Free Protein Analysis with Downstream Electrodes for Direct Removal of Electrolysis Products. Lab Chip 18, 162-170 (2018).

8. Buell, A. K. et al. Solution conditions determine the relative importance of nucleation and growth processes in a-synuclein aggregation. Proc. Natl. Acad. Sci. U. S. A. 111, 7671-7676 (2014).

9. Harding, S. E., Rowe, A. J. \& Horton, J. C. Analytical Ultracentrifugation in Biochemistry and Polymer Science. (1992).

10. Schuck, P. www.analyticalultracentrifugation.com/default.htm.

11. Turgeon, R. T., Fonslow, B. R., Jing, M. \& Bowser, M. T. Measuring Aptamer Equilbria Using Gradient Micro Free Flow Electrophoresis. Anal. Chem. 82, 3636-3641 (2010).

12. Horrocks, M. H. et al. Fast Flow Microfluidics and Single-Molecule Fluorescence for the Rapid Characterization of a-Synuclein Oligomers. Anal. Chem. 87, 8818-8826 (2015).

13. Kjaergaard, M. et al. Oligomer Diversity during the Aggregation of the Repeat Region of Tau. ACS Chem. Neurosci. 9, 3060-3071 (2018). 
14. Fonslow, B. R. \& Bowser, M. T. Optimizing band width and resolution in micro-free flow electrophoresis. Anal. Chem. 78, 8236-8244 (2006).

15. Andreasen, M., Lorenzen, N. \& Otzen, D. Interactions between misfolded protein oligomers and membranes: A central topic in neurodegenerative diseases? Biochim. Biophys. Acta Biomembr. 1848, 1897-1907 (2015).

16. Yates, E. V et al. Latent analysis of unmodified biomolecules and their complexes in solution with attomole detection sensitivity. Nat. Chem. 7, 802-809 (2015).

17. Doane, T. L., Chuang, C.-H., Hill, R. J. \& Burda, C. Nanoparticle -Potentials. Acc. Chem. Res. 45, 317-326 (2011).

18. Fischer, H., Polikarpov, I. \& Craievich, A. F. Average protein density is a molecular-weightdependent function. Protein Sci. 13, 2825-2828 (2004).

19. Tsukakoshi, K., Abe, K., Sode, K. \& Ikebukuro, K. Selection of DNA Aptamers That Recognize a-Synuclein Oligomers Using a Competitive Screening Method. Anal. Chem. 84, 5542-5547 (2012). 